\title{
Primary Angioplasty for the Treatment of Acute ST Elevated Myocardial Infarction: Single Centre Experience
}

\author{
F Begum, KN Khan, NAM Momenuzzaman, KM Sohail, SMZ Haque, AM Shafique, SK Mallick, \\ R Rahman, MM Amin, M Rahman, F Haque \\ Department of Cardiology, United Hospital limited, Dhaka.
}

Keywords:

Primary

angioplasty, ST elevation MI.

\begin{abstract}
:
Background: Worldwide primary angioplasty is a recommended strategy of reperfusion in patient with acute myocardial infarction as because it ensures reperfusion of the infarct-related vessels more than $90 \%$ where as, with thrombolytics it is only $60-70 \%$.
\end{abstract}

Methods: It is a retrospective observational study includes all patients treated with primary angioplasty at United Hospital from Between March 2007 to August 2010. Total 237 consecutive patients with acute myocardial infarction were treated with primary angioplasty were included. Those presented beyond 12 hours of onset of chest pain, in cardiogenic shock, resuscitate and intubated before the procedural were excluded from the study.

Results: Majority (76\%) of the patient were male, age was minimum 28 years and maximum 80 years, $41.5 \%$ were diabetics, $58.4 \%$ were hypertensive, $43.5 \%$ were dyslipidaemic, $17 \%$ were smoker, $29.3 \%$ with positive family history. Fifty seven percent patients presented with anterior MI, $42 \%$ with inferior $M I$ and $1 \%$ with lateral MI. Left anterior descending (LAD) is the most common vessel involved (S7\%), followed by Right coronary artery (RCA) $31 \%$, Left circumflex artery (LCD $8 \%$, Ramus $1.3 \%$ and Graft vessel 2.7\%. Our door to balloon time was minimum 23 min, maximum 184 min. We used drug eluting stents for most of the patients , GP IIbIIIa receptor Mockers used in 50\% cases and thrombus suction device were used when indicated. We faced complications like arrhythmias in $24 \%$ hypotension in $18 \%$, no flow or slow flow in $45 \%$, cardiac arrest in $3 \%$ and coronary perforation in 1. \%. Our overall survival was $97.9 \%$.

Conclusion: Primary angioplasty is a emerging area in the context 149-154of our country. Many of the new centers start this novel strategy which helps to save many lives. Primary angioplasty is feasible and safe method of reperfusion in patient with acute myocardial infarction in our center.

(Cardiovasc.j. 2011; 3(2): 149-154)

\section{Introduction:}

The in-hospital management of ST-elevation myocardial infarction (STEMI) has evolved significantly during the last decade, with particular emphasis on primary percutaneous coronary intervention (PCI) as the preferred treatment strategy when feasible. Primary Angioplasty defined as balloon angioplasty undertaken as the primary reperfusion strategy for STEMI without previous or concomitant fibrinolytic therapy. Prompt reperfusion of ischaemic myocardium is the major focus of acute treatment of patients with STEMI. Survival in Patients presenting with STEMI depends on early, complete, and sustained reperfusion of infarct-related artery. ${ }^{1-3}$ The use of thrombolytic therapy in acute myocardial infarction(AMI) reduces mortality rates and improved left ventricular function. ${ }^{2,12}$ Pharmacological therapy with fibrinolytic agents can achieve early reperfusion, complete flow is restored at best in only $60 \%$ of patients. ${ }^{4}$ The benefits of thrombolytic therapy are limited by re-occlusion of the infarct-related artery, which occurs in 25\% to $30 \%$ of patients after successful reperfusion ${ }^{3,13}$ Infarct artery reocclusion after AMI increases morbidity and mortality rates and blunts myocardial salvage ${ }^{14}, 15$ Mechanical reperfusion with primary percutaneous intervention(PCI) can restore complete flow in up-to $95 \%$ of patients and is associated with a lower rate of reocclusion. ${ }^{4}$ There is no question that primary PCI, when available is the treatment of choice. ${ }^{5}$ In our hospital we have primary angioplasty program and we are doing 24 hours a day and 365 days a year.

\section{Rationale to do Primary PCI:}

Fibrinolytic therapy has been an important means of establishing reperfusion for decades. However, 
limitations to the use of thrombolytic therapy include perceived or definite contraindications(15$20 \%)$, intracranial bleeding(1-2\%), inability to establish TIMI-3 flow in many patients(40\%) and high rates of recurrent ischaemia and reocclusion (25-30\%). ${ }^{6}$ Accordingly, Primary PCI has emerged as the preferred reperfusion strategy.

\section{Advantage of Primary PCI:}

- High patency rates ( $>95 \%$ ) with brisk ante grade flow

- Lower mortality

- Better residual left ventricular function

- More rapid electrocardiographic normalization

- Less recurrent ischaemia (angina, reinfarction, exercise induced ischemia)

- No systemic fibrinolysis, therefore bleeding problems avoided

- Improved risk stratification by angiography with identification of patients suitable for coronary artery by pass surgery

\section{Disadvantages of Primary PCI}

- Higher procedural cost than streptokinase or alteplase (although long term costs lower)

- Can be performed only when cardiac catheterization facilities and experienced staff available

- Recanalisation more rapid than thrombolysis only if 24 hour on-call team available

- Risks and complications of cardiac catheterization and percutaneous intervention

- Reperfusion arrhythmias probably more common because of more rapid recanalisation

The morbidity and mortality associated with primary angioplasty is operator dependant, varying with the skill and experience of interventionalist. ${ }^{6}$. For that reason ACC/AHA recommend some criteria to perform primary PCI.

\section{Methods and Patients:}

Retrospective, observational study including all patients presenting with STEMI within 12 hours of onset of chest pain and underwent primary PCI as a reperfusion strategy at united hospital from March 2007-August 2010.
Operator, Laboratory and institutional requirement:

We established the followings standards for the performance of primary angioplasty for operators, laboratories and institutions at our hospital.

1. Operators are experienced interventionalist who regularly performs intervention.

2. The nursing and technical catheterization laboratory staffs are well experienced to handle acutely ill patients and comfortable with interventional equipments.

3. The catheterization laboratory itself is well equipped with option of resuscitative equipment and intra-aortic balloon pump (IABP) support.

4. The cardiac care unit nurses are well trained in hemodynamic monitoring of the patients

5. Hospital administration fully supports the Primary PCI program.

6. Written consent must be taken from the patients or patient's relative.

7. We have ongoing program to analysis outcomes.

\section{Study population:}

Inclusion criteria-

1. Patient presented with chest pain, ECG changes suggestive of STEMI

2. Duration of pain $<12$ hours

3. All age group

4. Both sexes

5. Underwent Primary PCI as a reperfusion strategy

\section{Exclusion criteria}

1. patient presented with cardiogenic shock

2. Chest pain $>12$ hours

Medications and technique:

All patients got aspirin $300 \mathrm{mg}$ and $600 \mathrm{mg}$ clopidogrel, GTN-oral/IV, 5000units IV heparin at Emergency Department immediacy after diagnosis. In Cath lab 10,000 units IV Heparin before initiation of PCI was given, some times more 
heparin needed to keep ACT .300. We used intracoronary GTN, Adenosine if there was slow flow or no flow. IV GPIIb/IIIa receptor blockers bolus followed by IV infusion no flow or slow or huge thrombus burden. We did not use distal protection device. Following PCI 3-6 doses of LMWH subcutaneously given routinely if there is no bleeding episodes. Introducing sheath were removed 2 hours after completion of GPIIb/IIIa receptor blockers or 6 hours after completion of the procedure.

From emergency patients were shifted directly to the cath-lab. Both arterial and venous femoral access was achieved immediately. TPM was implanted if bradycardia or heart block present at presentation. We used aspiration thrombectomy catheter before ballooning if there was thrombus burden. Pre-dilatation with balloon was done if lesion morphology were complex and critical after thrombus aspiration. In our protocol we did angioplasty to the infarct related artery then staged PCI or CABG. Most of the cases we put DES stents except when clinical condition demand BMS. We routinely did post dilatation after stent implantation.

\section{Results:}

Total 237 patients were included in this study From March 2007 to August 2010. Minimum age included was 28 years, maximum $80 y$ rs with mean age 55.8 \pm 11.5 . Majority of the patients were male $76 \%$. Risk factor analysis showed $58.4 \%$ were hypertensive, $41.5 \%$ diabetics, $43.5 \%$ dyslipidaemic, $29.3 \%$ current smoker, $17 \%$ with positive family history of coronary artery disease. Around two third of the procedure (61\%) were performed during off time (6pm to 9am). Among patients $57 \%$ presented with Anterior MI, $42 \%$ with inferior MI. and $1 \%$ with lateral MI. At our hospital door to needle time was minimum 23 minutes, maximum 180 minutes with average 84 minutes. Single vessel diseases were found most of the cases (54.8\%), double vessels disease were $(28.6 \%)$ and $16.7 \%$ having triple vessel disease. LAD was the culprit vessel in 57\% cases, RCA $31 \%$, LCX was $8 \%$. Heavy thrombus burden was seen $85 \%$ cases. $85 \%$ cases culprit vessels were totally occluded with TIMI 0 distal flow rest of the cases $95-99 \%$ occlusion with TIMI I distal flow. Thrombus suction catheters were used $90 \%$ of the procedures. $91.8 \%$ of the procedure we preferred drug eluting stents. During procedure arrhythmias( VT, SVT, AF, CHB)-24\%, hypotension $16 \%$ need inotrope support, $2 \%$ IABP support, No flow/ slow flow 45\%, cardiac arrest needs CPR $3 \%$, coronary artery perforation $1 \%$, cardiac tamponade 1\%. Complications were managed accordingly. No patient required emergency bypass surgery as a result of cath -lab complications. Survival rate were $97.9 \%$. Among 5 patients, 2 died due to cardiac arrest in CCU, Cardiac arrest developed those patients having triple vessel disease. 3 patients have to put on artificial ventilator, died due to multi system involvement as a result of septicemia. After procedure during CCU stay we did bed side echocardiogram $82.5 \%$ patients maintaining EF > $50 \%, 10.8 \%$ of patients $45-49 \%$ and $6.5 \%$ patients $35-45 \%$.

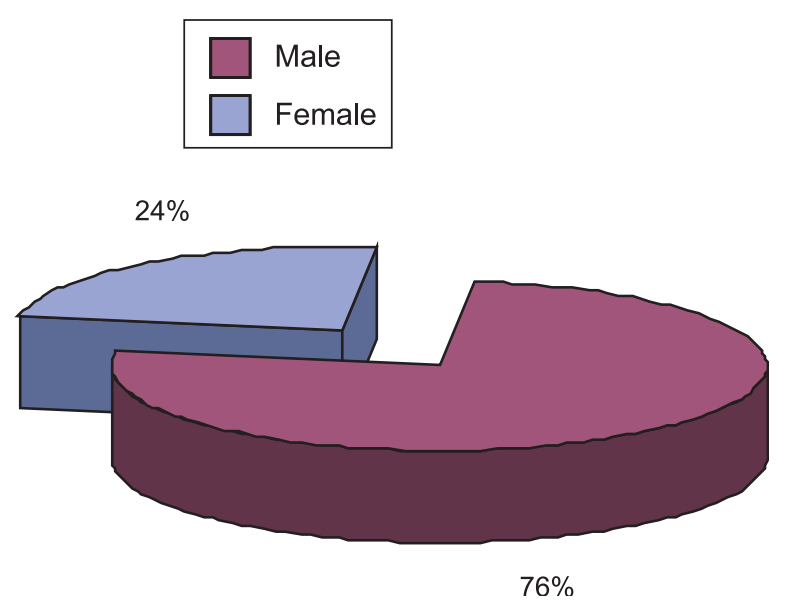

Fig.-1: Male female distribution

Table-I

Age distribution

\begin{tabular}{lc}
\hline Age & $\%$ \\
\hline Mean & $55.8 \pm 11.5$ \\
$<40 y r s$ & 5.6 \\
$40-49 y r s$ & 26.8 \\
$50-59 y r s$ & 36.5 \\
$60-69 y r s$ & 17.2 \\
$>70 y r s$ & 15.2 \\
\hline
\end{tabular}




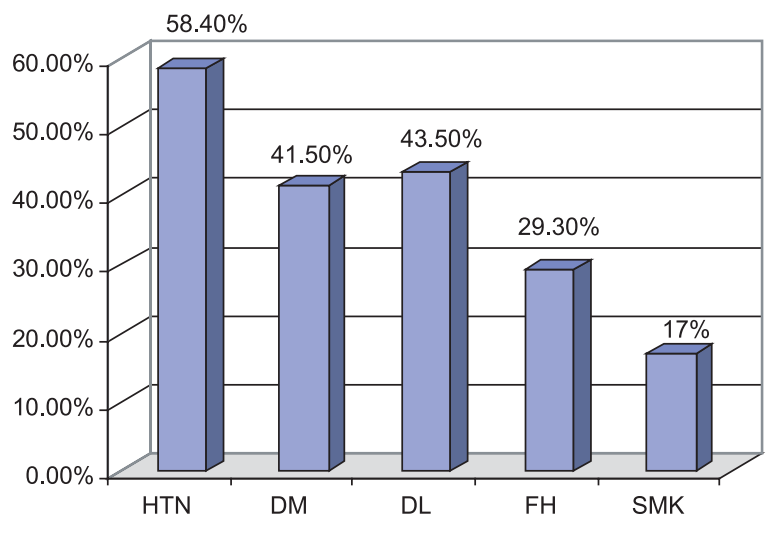

Fig.-2: Risk factor distribution.

Table-III

Angiographic pattern

\begin{tabular}{lc}
\hline No of Vessel involvement & $\%$ \\
\hline SVD & 54.8 \\
DVD & 28.6 \\
TVD & 16.7 \\
\hline
\end{tabular}

Table-IV

Stents characteristics

\begin{tabular}{lc}
\hline Stents & $\%$ \\
\hline DES & 87.8 \\
BMS & 12.2 \\
\hline
\end{tabular}

\section{Off-time}

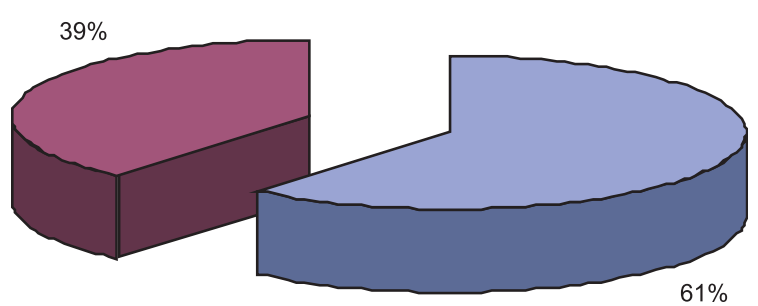

Fig.-3: Time distribution of Primary angioplasty. Office hour ( 9 am to $6 \mathrm{pm}$ ) Off-time ( $6 \mathrm{pm}$ to 9 am)
Table-V

Complications of Primary angioplasty

Asystole or heart block

$4.1 \%$

requiring pacemaker

$\mathrm{VF}$

$2.7 \%$

Hypotension

$45.7 \%$

Pulmonary oedema

$2 \%$

Cardiogenic shock

$5.4 \%$

Respiratory arrest

$1.5 \%$

Aspiration

$0.8 \%$

Anaphylaxis

$0 \%$

$\mathrm{ARF}$

$0.6 \%$

Stroke or TIA

$0 \%$

Vascular occlusion / Pseudo aneurysm

$0.8 \%$

Coronary perforation

$0.8 \%$

Pericardial tamponade

$0.2 \%$.

Emergency CABG

0

Death

$2.1 \%$

Death due to cath lab complication

Table-VI

In-Hospital Outcomes in patients undergoing primary angioplasty

\begin{tabular}{lc}
\hline Outcome & $\%$ \\
\hline Death & $2.1 \%$ \\
Reinfarction & $1.3 \%$ \\
Reocclusion & $2.5 \%$ \\
Stroke or TIA (non hemorrhagic) & 0 \\
\hline
\end{tabular}

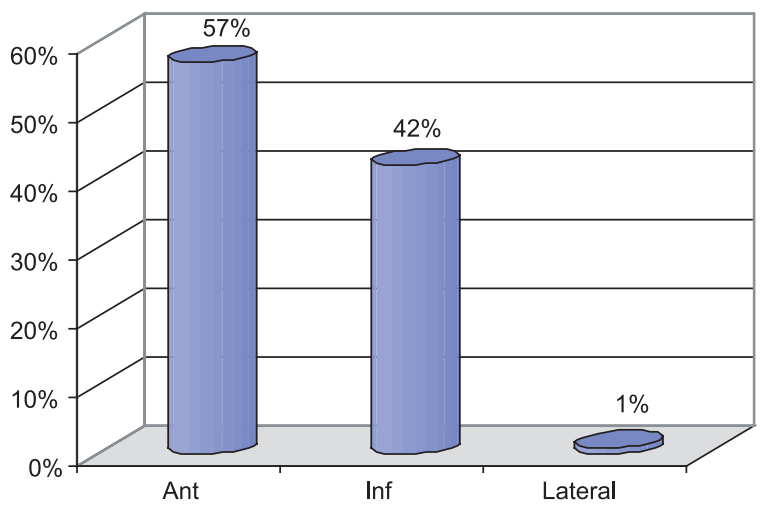

Fig 4: Distribution of MI (According to location) in the study population. 


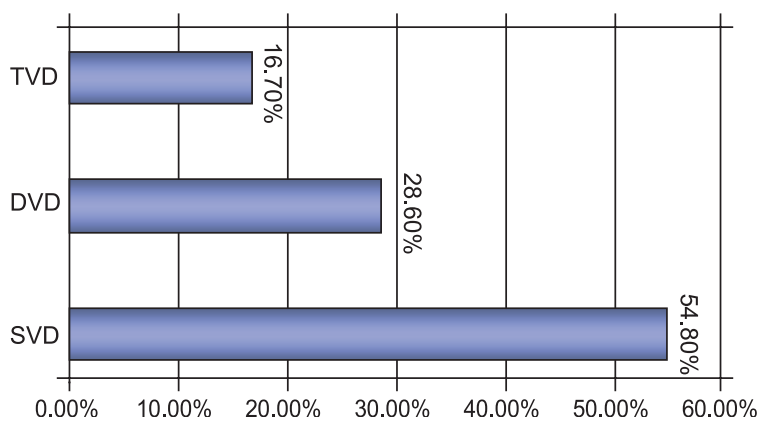

Fig 5: Extent of disease in the study population.

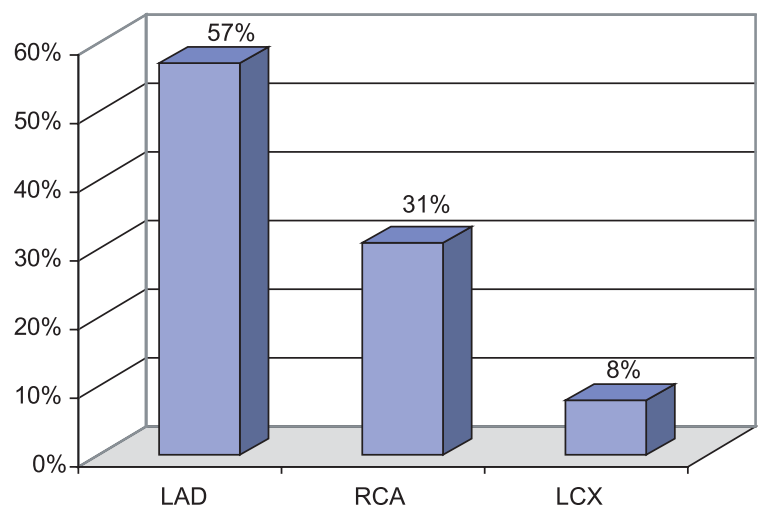

Fig 6: Distribution of Stent implantation according to location.

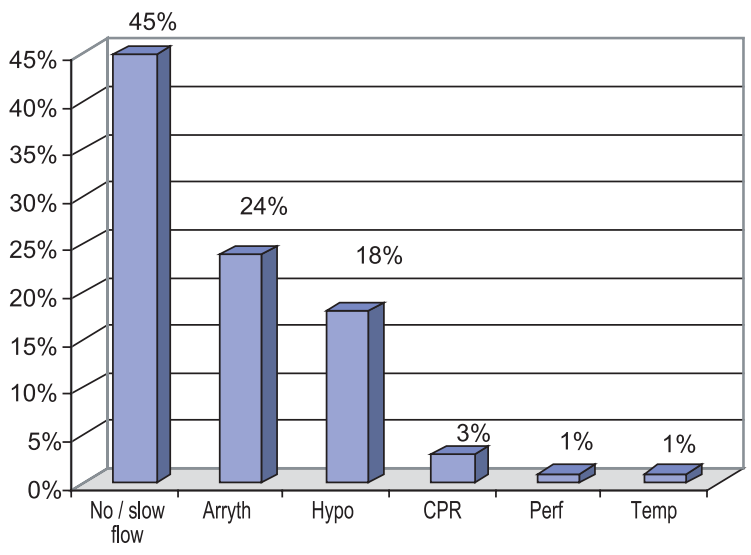

Fig 7: Complications during procedure.

\section{Discussion:}

There is no question that primary PCI, when available, is the treatment of choice. ${ }^{7}$ But in our country it is not a widely used reperfusion strategy due to lack facilities. Only a few centers at Dhaka city are performing primary PCI but mostly during the office hours. But in our centre we have Primary
PCI facilities 24 hours a day, 365days a year. Study population was mostly male like all over the world. Regarding age we had younger age group, mean age $55.8 \pm 11.5 y r s$, another study at USA showing their mean age $61 \pm .13 y r s .{ }^{8}$ Lowest age in our series was 28yrs. Risk factors analysis showed HTN is the most common it was $58.4 \%$, it is also like other studies. A study at USA described HTN as the most common risk factor. ${ }^{8}$ Primary PCI holds a survival advantage if it can be performed in a timely fashion. The principle that "time is myocardium" applies to both fibrinolysis(door to needle $)^{11}$ and primary PCI(door to balloon) ${ }^{9}$ Mortality rate is similar with primary PCI when compared with SK for patients presenting within 3hours of onset of symptoms but lower with PCI in those presenting after 3 hours. ${ }^{11}$ In our hospital mean door to needle time was 84 minutes, minimum 23minutes and maximum180minutes. Our door to needle time is internationally comparable. In certain European countries door to needle time is under 30minutes on arrival at PCI centre, such rapid implementation of a Primary PCI strategy has not been accomplished to date in United states, where the median time from arrival at door of a referring hospital to balloon inflation time at a PCI centre after transfer is 185 minutes. ${ }^{10}$ In most of the cases we used aspiration thrombectomy devices according to ACC/ AHA guideline updated 2009, aspiration thrombectomy is reasonable for patients undergoing primary PCI. In most of the cases we used DES, ACC/ AHA guideline recommended DES as an alternative of BMS( ACC/AHA recommendation-2009). Our complications are like those of other studies, no patients needed emergency CABG as a part of cath lab complication. Our procedural successes were $99 \%$ and over all survival rate is $97.9 \%$. We excluded patients presented with cardiogenic shock and $>12$ hours from the onset of chest pain from the study. Cardiogenic shock is the definite indication for primary PCI but we excluded because of high mortality. We planned to study this group in separate for more details and critical analysis.

\section{Conclusion:}

Primary angioplasty is the recommended way of revascularization in STEMI if facilities are available. Time is most important factors to save myocardium as well as outcome of the patients. 
Our initial experiences are encouraging and help us to establish PAMI as an alternative to thrombolytics to those who can afford and needs most. Most of our patients are self referred. Awareness regarding PAMI among physicians and cardiologists will help the patients to improve immediate and long term outcome after STEMI.

\section{References:}

1. Effectiveness of intravenous thrombolytic treatment in acute myocardial infarction. Gruppo Italiono per lo studiolo della Streptochinasi nell'Infarto Miocardioco (GISSI). Lancet 1986;1:397-402.

2. The effects of tissue plasminogen activator, streptokinase, or both on coronary-artery patency, Venticular function, and survival after acute myocardial infarction. The GUSTO Angiographic Investigators [published erratum appears in N Eng J Med. 1994; 330:516]. N Eng J Med 1993; 329:1615-1622.

3. Ohman EM, Callif RM, Topol EJ, et al. Consequences of reocclusion after successful reperfusion therapy in acute myocardial infarction. TAMI study group. Circulation1990;82:781-791.

4. Gibson CM. Primary angioplasty compared with thrombolysis: new issues in the era of glycoprotein IIb/ IIIa inhibition and intra coronary stenting. Ann Intern Med 1999; 130:841-847.

5. Widimsky P, Groch L, Zelizko M, et al. Multicenter randomized trial comparing transport to primary angioplasty vs immediate thrombolysis vs combined strategy for patients with acute myocardial infarction presenting to a community hospital without a catheterization laboratory. The PRAGUE study. Eur Heart $J$ 2000;21:823-831

6. Ever D Grech, David R Ramsdale. Acute Coronary syndrome: ST segment elevation myocardial infarction. BMJ 2003; 326. 7403.137

7. Aversano T, Aversano LT, Passamani E, et al. Thrombolytic therapy vs primary percutaneous coronary intervention for myocardial infarction in patients presenting to hospitals without on-site cardiac surgery: a randomized controlled trial. JAMA. 2002; 287: 1943-1951.

8. Thomas P. Wharton, Nancy Sinclair Mc Namara, Frank A. Fedele, et al. Primary angioplasty for the treatment of acute myocardial infarction: experience at two community hospitals without cardiac surgery. $J$ Am Coll Cardiol 1999; 33:1257-1265.

9. Mc Namara RL, Wang Y, Herrin j, et al. Effect of door to needle time on mortality in patients with ST segment elevation myocardial infarction. J Am Coll Cardiol 2006;47:2180-2186.

10. National Registry of Myocardial Infarction-4. Quarterly Report. March 200. Dataon file. 2003.

11. Widimsky P, Budesinsky T, Vorac d, et al. Long distance transport for primary angioplasty VS immediate thrombolysis in acute myocardial infarction; final results of the randomized national multicentre trial: PRAGUE2national muticentre. Eur Heart J 2003;24: 94-104.

12. ISIS-2 (Second International study of Infarct Survival). Randomized trial of intravenous streptokinase, oral aspirin, both or neither among 17,187 cases of suspected acute myocardial infarction. Lancet 1988:349; 60.

13. Nijland F, Kamp O, Verheugt FWA, etal. Long- term implications of reocclusion on left ventricular size and function after successful thrombolysis for first anterior myocardial infarction. Circulation 1997;95:111-7

14. Meijer A, Vereugt FWA, Werter CJPJ,et al. Aspirin versus Coumadin in the prevention of reocclusion and recurrent ischaemia after successful thrombolysis: a perspective placebo-controlled angiographic study. Circulation 1993;87:1524-30

15. Takens BH, Brugeman J, Van der meer J, et al. Reocclusion three months after successful throbolytic treatment of acute myocardial infarction with anisoylated plasminogen streptokinase activatingcomplex. Am J Cardiol 1990; 65:1422-4. 